\title{
WEAKLY DENSE SUBSETS OF THE MEASURE ALGEBRA
}

\author{
MAXIM R. BURKE
}

(Communicated by R. Daniel Mauldin)

\begin{abstract}
We introduce the notion of the weak density of a Boolean algebra and show that for homogeneous measure algebras it coincides with the density (=least size of a coinitial set). From this we obtain a partial lifting of the measure algebra of $[0,1]$ of minimal size which does not extend to a lifting. It also follows that the $\pi$-character of each point and the $\pi$-weight are the same for the Stone space of a homogeneous measure algebra
\end{abstract}

\section{Notation}

For set-theoretic and topological notation not described below, see $[3,8,9$, 10]. For measure theory see [7]. If $B$ is a Boolean algebra and $a \in B, a^{c}$ is the complement of $a . B_{a}=B \mid a=\{b \in B: b \leq a\} . B$ is homogeneous if $B_{a}$ is isomorphic to $B$ for all $a \in B \backslash\{0\}$. If $f$ is a function which assigns an ordinal to each Boolean algebra, then $B$ is homogeneous in $f$ if $f\left(B_{a}\right)=f(B)$ for all $a \in B \backslash\{0\}$. St $(B)$ is the Stone space of $B$. If $B^{\prime} \subseteq B$ is a subalgebra and $a \in B$ then $\left\langle B^{\prime} \cup\{a\}\right\rangle=\left\{(b \wedge a) \vee(c \backslash a): c, b \in B^{\prime}\right\}$ is the subalgebra generated by $B^{\prime} \cup\{a\}$. For any infinite set $K,[K]^{\omega}$ is the partial order of countably infinite subsets of $K$, ordered by inclusion. $c f[K]^{\omega}=\min \left\{|D|: D \subseteq[K]^{\omega}\right.$ and $\left.\left(\forall B \in[K]^{\omega}\right)(\exists A \in D)(B \subseteq A)\right\}$.

If $(X, \Sigma, \mu)$ is a probability space and $A, B \in \Sigma$ then we write $A \subseteq^{*} B$, $A={ }^{*} B$ to mean $\mu(A \backslash B)=0, \mu(A \Delta B)=0$, respectively. If $I$ is any nonempty set, then $\left(X^{I}, \mu\right)$ is the product space with product measure denoted also by $\mu$. For $a \in I$ let $\pi_{a}: X^{I} \rightarrow X^{I \backslash\{a\}}$ be defined by $\pi_{a}(f)=\left.f\right|_{I \backslash\{a\}}$. Then for any set $E \subseteq X^{I}, \operatorname{supp}(E)=\left\{a \in I: E \neq \pi_{a}^{-1} \pi_{a}^{\prime \prime} E\right\}$.

For cardinals $\kappa \geq \omega, \mu$ is the usual product measure on $\{0,1\}^{\kappa}, \Sigma_{\kappa}$ is the $\sigma$-algebra of measurable sets, and $\mathscr{N}_{\kappa}=\left\{E \in \Sigma_{\kappa}: \mu E=0\right\} . \mathscr{A}_{\kappa}=\Sigma_{\kappa} / \mathscr{N}_{\kappa}$ is the measure algebra of $\left(\{0,1\}^{\kappa}, \Sigma_{\kappa}, \mu\right)$. If $E \in \Sigma_{\kappa}$ then $[E] \in \mathscr{A}_{\kappa}$ is the equivalence class of $E$. If $B$ is a subalgebra of $\mathscr{A}_{\kappa}$, then a partial lifting $\rho: B \rightarrow \Sigma_{\kappa}$ is a Boolean homomorphism satisfying $\rho(a) \in a$ for each $a \in B$. If $[U] \in B$, and $\rho[U] \supseteq U$ for each clopen Baire set $U$, then $\rho$ is called a

Received by the editors July 24, 1987 and, in revised form, September 14, 1988.

1980 Mathematics Subject Classification (1985 Revision). Primary 06E99, 28A60, 28A51, $54 \mathrm{~A} 25$. 
strong partial lifting. If $B=\mathscr{A}_{\kappa}, \rho$ is called a lifting. For finite functions $s$, let $[s]=\left\{f \in\{0,1\}^{\kappa}: s \subseteq f\right\}$; if $x \in E \in \Sigma_{\omega}$, the density of $E$ at $x$ is $d(x, E)=\lim _{n \rightarrow \infty} \mu(E \cap[x \mid n]) / \mu([x \mid n])$ when this limit exists. For almost all $x \in E, d(x, E)=1$ (see e.g. [12]).

\section{INTRODUCTION}

Definition. Let $B$ be a Boolean algebra. Then $D \subseteq B \backslash\{0\}$ is dense if $(\forall b \in$ $B \backslash\{0\})(\exists d \in D)(d \leq b) . \quad D$ is weakly dense if $(\forall b \in B \backslash\{0\})(\exists d \in D)(d \leq$ $b$ or $\left.d \leq b^{c}\right)$. Let $d(B)=\min \{|D|: D \subseteq B$ is dense $\}, \operatorname{wd}(B)=\min \{|D|: D \subseteq$ $B$ is weakly dense $\}$.

When $B=\mathscr{A}_{\kappa}$ for some $\kappa$, we will say $D \subseteq \Sigma_{\kappa}$ is dense-(weakly dense) in $\mathscr{A}_{\kappa}$ if $\{[d]: d \in D\}$ is dense (weakly dense) in $\mathscr{A}_{\kappa}$.

Remarks. (1) $\operatorname{wd}(B) \leq d(B)$.

(2) For any $a \in B \backslash\{0\}$, if $D \subseteq B_{a}$ is dense or weakly dense in $B_{a}$, then $D$ is weakly dense in $B$. Thus if $B$ is not homogeneous in $d$ then $\operatorname{wd}(B)<d(B)$.

(3) If $B$ is $\sigma$-complete and homogeneous in $d$ and $d(B) \geq \omega_{1}$ then $\operatorname{wd}(B)$ $\geq \omega_{1}$. (Proof: Note that the hypotheses imply that $B$ is nonatomic. If $\left\{a_{n}: \eta \in\right.$ $\omega\} \subseteq B \backslash\{0\}$ is any countable set, inductively choose disjoint elements $b_{n} \in B$ satisfying $b_{n} \subseteq a_{n}$ and $a_{i} \backslash \bigvee_{j=1}^{n} b_{j} \neq 0 \quad(i>n)$. (To choose $b_{n}$ note that $D=\left\{a_{i} \wedge a_{n} \backslash \bigvee_{j=1}^{n-1} b_{j}: i>n\right\}$ is not dense in $B_{a_{n}}$ and choose $b_{n} \in B_{a_{n}} \backslash\{0\}$ which does not contain any member of $D$. This works.) Now write $b_{n}=c_{n} \vee d_{n}$, $c_{n} \neq 0 \neq d_{n}, c_{n} \wedge d_{n}=0$. Let $d=\sup \left\{d_{n}: n \in \omega\right\}$. For each $n$, both $d \wedge a_{n}$ and $d^{c} \wedge a_{n}$ are not 0 and hence $\left\{a_{n}: n \in \omega\right\}$ is not weakly dense.)

(4) If $\mathscr{A}$ is any measure algebra, $\{x \in \mathscr{A}: \mu(x)=1 / 2\}$ is weakly dense in $\mathscr{A}$.

(5) If $U$ is a local $\pi$-base on $B$ (i.e. $U \subseteq B \backslash\{0\}$ and for some ultrafilter $p \subseteq B \backslash\{0\},(\forall b \in p)(\exists a \in U)(a \leq b))$, then $U$ is weakly dense in $B$. Since $\pi \chi(x, \operatorname{St}(B)) \geq \operatorname{wd}(B)$ for each $x \in \operatorname{St}(B)$, if $\operatorname{wd}(B)=d(B)$ then $\pi \chi(x, \operatorname{St}(B))=\pi w(\operatorname{St}(B))$ for all $x \in \operatorname{St}(B)$. (For any topological space $X$ and for any $x \in X, \pi w(X)=\min \{|\mathscr{A}|: \mathscr{A}$ is a collection of nonempty open sets such that every nonempty open set includes a member of $\mathscr{A}\}$, and $\pi \chi(x, X)=\min \{|\mathscr{A}|: \mathscr{A}$ is a collection of nonempty open sets such that every neighborhood of $x$ includes a member of $\mathscr{A}\}$. For more on these cardinal functions see [ 3 or 8$]$.)

(6) If $\kappa$ is given the discrete topology and we let $B(\kappa)$ be the clopen subsets of $\kappa^{\omega}$ (the Baire space of length $\kappa$ ), then $d(B(\kappa))=\kappa$ and $\operatorname{wd}(B(\kappa))=\omega$ (a countable neighborhood base at any point is weakly dense). However in the completion of $B(\kappa)$ the weak density is $\kappa$. (We omit the proof. It is similar to that of remark (3) above.)

Problem. Is there a homogeneous complete Boolean algebra $B$ such that $\operatorname{wd}(B)<d(B) ?$ 
In $\S 2$ of this paper we prove some lemmas that will be needed later on. In $\S 3$ we will show that for any $\kappa \geq \omega, \operatorname{wd}\left(\mathscr{A}_{\kappa}\right)=d\left(\mathscr{A}_{\kappa}\right)=d\left(\mathscr{A}_{\omega}\right) \cdot c f[\kappa]^{\omega}$. (The second equality is obtained in [5, Theorem 16] by different methods.) In $\S 4$ we give an application in the theory of liftings.

\section{Preliminary lemmas}

Lemma 1. If $(X, \lambda),(Y, \mu),(Z, \nu)$ are probability spaces and if $A \subseteq X \times Y$, $B \subseteq Y \times Z$ are measurable sets which satisfy $A \times Z \subseteq^{*} X \times B$, then there is a measurable set $C \subseteq Y$ such that

$$
A \times Z \subseteq^{*} X \times C \times Z \subseteq^{*} X \times B .
$$

Proof. For $x \in X$ and $z \in Z$ define $A_{x}=\{y \in Y:(x, y) \hat{\epsilon} A\}, B_{z}=\{y \in$ $Y:(y, z) \in B\}$. If $\chi_{A}(x, y), \chi_{B}(y, z)$ are the characteristic functions of $A, B$ respectively, then we have $\chi_{A}(x, y) \leq \chi_{B}(y, z)$ for a.a. $(x, y, z) \in X \times Y \times Z$. By Fubini's theorem this means that for a.a. $(x, z)$, for a.a. $y, \chi_{A}(x, y) \leq$ $\chi_{B}(y, z)$. Thus for a.a. $z$,

$$
\text { for a.a. } x, A_{x} \subseteq^{*} B_{z} \text {. }
$$

Let $C$ be a set of minimal measure s.t.

$$
\text { for a.a. } x, A_{x} \subseteq \subseteq^{*} C \text {. }
$$

$(\mathscr{C}=\{C:$ (2) holds for $C\}$ is closed under countable intersections. So for example choose $C_{n} \in \mathscr{C}$ s.t. $C_{0} \supseteq C_{1} \supseteq \cdots$ and $\mu C_{n} \searrow \inf _{C \in \mathscr{C}} \mu C$. Take $C=\bigcap_{n} C_{n}$.)

By minimality of $\mu C$, for any $z$ satisfying (1), $C \subseteq^{*} B_{z}$, which together with (2) implies $A \times Z \subseteq^{*} X \times C \times Z \subseteq^{*} X \times B$.

Lemma 2. Let $(X, \mu)$ be a nonatomic probability space. Then there is a measurable subset $E \subseteq X^{\omega}$ s.t. for any $D$ of positive measure satisfying either $D \subseteq \subseteq^{*} E$ or $D \subseteq^{*} E^{c}, \operatorname{supp}(D)=\omega$.

Proof. Fix a sequence of measurable sets $E_{n} \subseteq X(n \geq 1)$ s.t. $0<\mu E_{n}<1$ and $\prod_{n \geq 1} \mu E_{n}>0$. Inductively define $A_{n} \subseteq X^{n}(n \geq 1)$ by

$$
\begin{gathered}
A_{1}=E_{1}, \\
A_{n+1}=\left(A_{n} \times E_{n+1}\right) \cup\left(A_{n}^{c} \times E_{n+1}^{c}\right) .
\end{gathered}
$$

By induction on $n \geq 1$ we have

(3) For all $m<n, A_{m} \times \prod_{m<i \leq n} E_{i} \subseteq A_{n}$ and $A_{m}^{c} \times \prod_{m<i \leq n} E_{i} \subseteq A_{n}^{c}$.

(4) For every $D \subseteq X^{n}$ s.t. $\mu D>0$ and $\operatorname{supp}(D) \neq n$ we have $\mu\left(D \cap A_{n}\right)>0$ and $\mu\left(D \cap A_{n}^{c}\right)>0$.

Proof. (3) is easy. For (4): when $n=1$ only $D=X$ satisfies the assumptions. Assume (4) and suppose $D \subseteq X^{n+1}, \mu D>0, \operatorname{supp}(D) \neq n+1$. 
Case 1. $n \notin \operatorname{supp}(D)$. Write $D=D^{\prime} \times X, D^{\prime} \subseteq X^{n}$. One of the sets

$$
\begin{aligned}
& D \cap\left(A_{n} \times E_{n+1}\right)=\left(D^{\prime} \cap A_{n}\right) \times E_{n+1}, \\
& D \cap\left(A_{n} \times E_{n+1}^{c}\right)=\left(D^{\prime} \cap A_{n}\right) \times E_{n+1}^{c}, \\
& D \cap\left(A_{n}^{c} \times E_{n+1}\right)=\left(D^{\prime} \cap A_{n}^{c}\right) \times E_{n+1}, \\
& D \cap\left(A_{n}^{c} \times E_{n+1}^{c}\right)=\left(D^{\prime} \cap A_{n}^{c}\right) \times E_{n+1}^{c},
\end{aligned}
$$

must have positive measure. Thus the first two of these sets or the last two both have positive measure. Either way $\mu\left(D \cap A_{n+1}\right)>0$ and $\mu\left(D \cap A_{n+1}^{c}\right)>0$.

Case 2. For some $k<n, k \notin \operatorname{supp}(D)$. One of $D \cap\left(X^{n} \times E_{n+1}\right), D \cap\left(X^{n} \times\right.$ $\left.E_{n+1}^{c}\right)$ has positive measure, say $\mu\left(D \cap\left(X^{n} \times E_{n+1}\right)\right)>0$. (The argument ior the other case is obtained by replacing $E_{n+1}$ by $E_{n+1}^{c}$ in what follows.) For $e \in E_{n+1}$, write $D_{e}=\left\{x \in X^{n}:(x, e) \in D\right\}$.

Let $S=\left\{e \in E_{n+1}: \mu D_{e}>0\right\}$. Then $\mu S>0$. Since $\operatorname{supp}\left(D_{e}\right) \neq n$, by the induction hypothesis, for $e \in S, \mu\left(D_{e} \cap A_{n}\right)>0$ and $\mu\left(D_{e} \cap A_{n}^{c}\right)>0$. Thus $\mu\left(D \cap\left(A_{n} \times E_{n+1}\right)\right)_{e}>0$ for all $e \in S$ and so $\mu\left(D \cap A_{n+1}\right)>0$, and similarly $\mu\left(D \cap A_{n+1}^{c}\right)>0$.)

Now let $E=\bigcup_{n}\left(A_{n} \times \prod_{i>n} E_{i}\right) \subseteq X^{\omega}$. Fix $m \geq 1$. Then for each $n>m$, by (1) we have $A_{m}^{c} \times \prod_{m<i \leq n} E_{i} \subseteq A_{n}^{c}$ and so $\left(A_{m}^{c} \times \prod_{i>m} E_{i}\right) \cap\left(A_{n} \times \prod_{i>n} E_{i}\right)=\varnothing$. Similarly $\left(A_{m} \times \prod_{i>m} E_{i}\right) \cap\left(A_{n}^{c} \times \prod_{i>n} E_{i}\right)=\varnothing$. Hence

$$
A_{n}^{c} \times \prod_{i>n} E_{i} \subseteq E^{c} \quad \text { for all } n \geq 1 .
$$

To finish the proof, suppose $D \subseteq X^{\omega}, \mu D>0$, and for some $k \in \omega$, $k \notin \operatorname{supp}(D)$. We must show $\mu(D \cap E)>0$ and $\mu\left(D \cap E^{c}\right)>0$. Since $\mu\left(X^{n} \times \prod_{i>n} E_{i}\right) \rightarrow 1$ as $n \rightarrow \infty$, there is an $n>k$ s.t. $D \cap\left(X^{n} \times \prod_{i>n} E_{i}\right)$ has positive measure. Note that $k \notin \operatorname{supp}\left(D_{e}\right)$, where $D_{e}=\left\{x \in X^{n}:(x, e) \in D\right\}$ for $e \in \prod_{i>n} E_{i}$. Let $S=\left\{e \in \prod_{i>n} E_{i}: \mu D_{e}>0\right\}$. Then $\mu S>0$ and for $e \in S$, by (4) we have $\mu\left(D_{e} \cap A_{n}\right)>0$ and $\mu\left(D_{e} \cap A_{n}^{c}\right)>0$. Since $D_{e} \cap A_{n}=\left(D \cap\left(A_{n} \times \prod_{i>n} E_{i}\right)\right)_{e}, \mu\left(D \cap\left(A_{n} \times \prod_{i>n} E_{i}\right)\right)>0$ and similarly $\mu\left(D \cap\left(A_{n}^{c} \times \prod_{i>n} E_{i}\right)\right)>0$; by (5) and the definition of $E$ these give $\mu(D \cap$ $\left.E^{c}\right)>0$ and $\mu(D \cap E)>0$ respectively.

Remark. The assumption that $X$ is nonatomic is needed here. For example if $X=\{0,1\}, \mu\{0\}=\mu\{1\}=1 / 2$, then for any $E \subseteq X^{\omega}$ s.t. $\mu E>0$, there is a $D \subseteq E$ s.t. $\mu D>0$ and $\omega \backslash \operatorname{supp}(D)$ is infinite.

Proof. The following proof is a simplification due to D. Fremlin of a proof which appeared in an earlier draft of this paper.

Let $E \subseteq X^{\omega}, \mu E>0$. For each $I \in[\omega]^{<\omega}$, define $f_{I}: 2^{\omega} \rightarrow 2^{\omega}$ by $f_{I}(x)(n)=x(n)$ if $n \notin I$, and $1-x(n)$ if $i \in I$. We write $f_{n}$ for $f_{\{n\}}$. Since $E$ can be approximated arbitrarily well by clopen sets $C$ (in the sense of making $\mu(E \Delta C)$ small) and for each clopen set $C, f_{n}^{\prime \prime}(C)=C$ for sufficiently large 
$n$, we have $\lim _{n \rightarrow \infty} \mu\left(E \Delta f_{n}^{\prime \prime}(E)\right)=0$. Inductively define a increasing sequence $\left\langle a_{n}: n \in \omega\right\rangle$ of elements of $\omega$ so that $D_{n}=\bigcap\left\{f_{I}^{\prime \prime}(E): I \subseteq\left\{a_{0}, \ldots, a_{n-1}\right\}\right\}$ has measure $\geq \mu(E) \cdot \prod_{i<n}\left(1-1 / 2^{i+1}\right)$. Then $D=\bigcap_{n<\omega} D_{n}=\bigcap\left\{f_{I}^{\prime \prime}(E): I \in\right.$ $\left.\left[\left\{a_{i}: i<\omega\right\}\right]^{<\omega}\right\}$ is as desired.

Lemma 3. If $N \in \mathscr{N}_{\omega}$, there is an $E \in \Sigma_{\omega}$ s.t.

$$
\{x: d(x, E)=1\} \cup\left\{x: d\left(x, E^{c}\right)=1\right\}
$$

is disjoint from $N$.

Proof. See [6, Theorem 4, p. 175].

\section{WEAK DENSITY OF MEASURE ALGEBRAS -}

Theorem 1. If $\kappa \geq \omega$ then $\operatorname{wd}\left(\mathscr{A}_{\kappa}\right)=d\left(\mathscr{A}_{\kappa}\right)=d\left(\mathscr{A}_{\omega}\right) \cdot c f[\kappa]^{\omega}$.

Proof. Case 1. $\kappa=\omega$. We must show $\operatorname{wd}\left(\mathscr{A}_{\omega}\right)=d\left(\mathscr{A}_{\omega}\right)$. This is similar to the proof in [2] that $d\left(\mathscr{A}_{\omega}\right)=$ cofinality of $\mathscr{N}_{\omega}$ (ordered by inclusion). Let $D \subseteq \Sigma_{\omega}$ of size $\operatorname{wd}\left(\mathscr{A}_{\omega}\right)$ be weakly dense in $\mathscr{A}_{\omega}$. It will suffice to find a cofinal set $\mathscr{B} \subseteq \mathscr{N}_{\omega}$ of size $|D|$. Without loss of generality assume that each $H \in D$ has density 1 at all its points. Let $Q$ be a countable dense subset of $\{0,1\}^{\omega}$. Take $\mathscr{B}=\left\{\left(\bigcup_{q \in Q} H+q\right)^{c}: H \in D\right\}$.

Let $N \in \mathscr{N}_{\omega}$. Find $H \in D$ s.t. $N_{1}=\bigcup_{q \in Q} N+q$ is disjoint from $H$. (If $E$ is as in Lemma 3 for $N_{1}$, then there is an $H \in D$ s.t. $H \subseteq^{*} E$ or $H \subseteq^{*} E^{c}$. Since $H$ has density 1 at all its points $H \cap N_{1}=\varnothing$.) For each $q \in Q$, we have $H \cap(N+q)=\varnothing$, or equivalently $N \cap(H+q)=\varnothing$. Thus $N \subseteq\left(\bigcup_{q \in Q} H+q\right)^{c} \in \beta$.

Case 2. $\kappa>\omega$. For each $A \in[\kappa]^{\omega}$ fix an enumeration $A=\{A(n): n \in \omega\}$ of $A$ and define $f_{A}:\{0,1\}^{\kappa} \rightarrow\{0,1\}^{\omega}$ by $f_{A}(x)(n)=x(A(n))$.

If $\mathscr{E} \subseteq \Sigma_{\omega}$ is dense in $\mathscr{A}_{\omega}$ and if $\mathscr{F} \subseteq[\kappa]^{\omega}$ is cofinal, then it is easy to check that $\left\{f_{A}^{-1}(E): A \in \mathscr{F}, E \in \mathscr{E}\right\}$ is dense in $\mathscr{A}_{\kappa}$. This shows that $d\left(\mathscr{A}_{\kappa}\right) \leq d\left(\mathscr{A}_{\omega}\right) \cdot c f[\kappa]^{\omega}$. There remains to show that $\operatorname{wd}\left(\mathscr{A}_{\kappa}\right) \geq d\left(\mathscr{A}_{\omega}\right) \cdot c f[\kappa]^{\omega}$.

Let $\mathscr{C}_{\kappa} \subseteq \Sigma_{\kappa}$ be a collection of Baire sets which is weakly dense in $\mathscr{A}_{\kappa}$. Define a family $\mathscr{E}_{\omega} \subseteq \Sigma_{\omega}$ as follows: If $E \in \mathscr{E}_{\kappa}$, let $C(E) \in \Sigma_{\omega}$ be a set of least measure satisfying $E \subseteq^{*} C(E) \times\{0,1\}^{\kappa \backslash \omega}$. Let $\mathscr{E}_{\omega}=\left\{C(E): E \in \mathscr{E}_{\kappa}\right\}$. Then $\left|\mathscr{E}_{\omega}\right| \leq\left|\mathscr{E}_{\kappa}\right|$ and $\mathscr{E}_{\omega}$ is weakly dense in $\mathscr{A}_{\omega}$. (Proof. If $F \in \Sigma_{\omega}, \mu F>0$, then there is an $E \in \mathscr{E}_{\kappa}$ s.t. $E \subseteq^{*} F \times\{0,1\}^{\kappa \backslash \omega}$ or $E \subseteq^{*} F^{c} \times\{0,1\}^{\kappa \backslash \omega}$, say the former. By definition of $C(E), C(E) \subseteq^{*} F$.) Thus $\operatorname{wd}\left(\mathscr{A}_{\kappa}\right) \geq \operatorname{wd}\left(\mathscr{A}_{\omega}\right)$. Now let $X=\{0,1\}^{\omega}$ and identify $\{0,1\}^{\kappa}$ with $X^{\kappa}$ via the map $f \mapsto\langle\langle f(\omega \cdot \alpha+n): n \in$ $\omega\rangle: \alpha\langle\kappa\rangle$. Now with $\mathscr{E}_{\kappa} \subseteq \Sigma_{\kappa}$ weakly dense in $\mathscr{A}_{\kappa}$, define a cofinal family $\mathscr{F} \subseteq[\kappa]^{\omega}$ by $\mathscr{F}=\left\{\operatorname{supp}(E): E \in \mathscr{E}_{\kappa}\right\}$ where $\operatorname{supp}(E)$ is taken in $X^{\kappa}$. To see that $\mathscr{F}$ is cofinal, fix $B \in[\kappa]^{\omega}$. By Lemma 2 , there is a measurable set $F \subseteq X^{B}$ s.t. for any measurable set $D \subseteq X^{B}$, if either $D \subseteq^{*} F$ or $D \subseteq \subseteq^{*} F^{c}$ then $\operatorname{supp}(D)=B$. Fix such an $F$ and let $E \in \mathscr{E}_{\kappa}$ be s.t. $E \subseteq^{*} F \times X^{\kappa / B}$ or $E \subseteq^{*} F^{c} \times X^{\kappa \backslash B}$, say the former. Claim: $B \subseteq \operatorname{supp}(E)$. For suppose 
$\alpha \in B \backslash \operatorname{supp}(E)$. Then there is a countable $S \subseteq \kappa$ and an $\bar{E} \subseteq X^{S}$ such that $\alpha \notin S$ and $E=\bar{E} \times X^{\kappa \backslash S}$. By Lemma 1, there is a measurable set $D \subseteq^{*} X^{B \cap S}$ s.t.

$$
E \subseteq^{*} D \times X^{\kappa \backslash(B \cap S)} \subseteq^{*} F \times X^{\kappa \backslash B} .
$$

Hence $D \times X^{B \backslash S} \subseteq^{*} F$; by the choice of $F, B \backslash S=\varnothing$, i.e. $B \subseteq S$, contradiction.

As mentioned in the introduction we have

Corollary. For each $x \in \operatorname{St}\left(\mathscr{A}_{\kappa}\right), \pi \chi\left(x, \operatorname{St}\left(\mathscr{A}_{\kappa}\right)\right)=\pi w\left(\operatorname{St}\left(\mathscr{A}_{\kappa}\right)\right)$.

(By a well-known theorem of D. Maharam [11a] every homogeneous measure algebra is isomorphic to $\mathscr{A}_{\kappa}$ for some $\kappa$.)

Remarks concerning the cardinal $c f[\kappa]^{\omega}$. (1) An easy induction on $n$ shows that $c f\left[\aleph_{n}\right]^{\omega}=\aleph_{n}$ for $n \in \omega$.

(2) If either $\mathrm{GCH}$ or the covering lemma with respect to an inner model of GCH holds then $c f[\kappa]^{\omega}=\kappa$ if $c f(\kappa)>\omega, \kappa^{+}$if $c f(\kappa)=\omega$. (See [9].)

(3) Assuming the consistency of large cardinals, there are models where $2^{\aleph_{0}}<$ $\aleph_{\omega}$ and $\aleph_{\omega}^{\aleph_{0}}>\aleph_{\omega+1}$. In any such model $c f\left[\aleph_{\omega}\right]^{\omega}>\aleph_{\omega+1}$. (See [11].)

\section{Partial Liftings}

When liftings are constructed inductively (see e.g. $[1,4])$ the problem arises of extending a partial lifting $\rho: B \rightarrow \Sigma_{\omega}$ to an element $a \in \mathscr{A}_{\omega} \backslash B$. In Theorem 2 below we compute the least size of a subalgebra $B$ for which such an extension can fail to exist.

Let $\lambda=\min \left\{|\mathscr{C}|: \mathscr{C} \subseteq \mathscr{N}_{\omega}\right.$ and $\left.\bigcup \mathscr{C} \notin \mathscr{N}_{\omega}\right\}$.

Lemma 4. $\operatorname{wd}\left(\mathscr{A}_{\omega}\right) \geq \min \left\{|A|: A \subseteq\{0,1\}^{\omega}\right.$ and $A$ has positive outer measure $\}$. In particular, $\operatorname{wd}\left(\mathscr{A}_{\omega}\right) \geq \lambda$.

Proof. Let $D \subseteq \Sigma_{\omega}$ be dense in $\mathscr{A}_{\omega},|D|=\operatorname{wd}\left(\mathscr{A}_{\omega}\right)$.

For each $E \in D$, choose a point $x(E) \in E$ of density 1 . Then $A=$ $\{x(E): E \in D\}$ has outer measure 1 .

Theorem 2. There is a subalgebra $B \subseteq \mathscr{A}_{\omega}$ of size $\lambda$ as well as a strong partial lifting $\rho: B \rightarrow \Sigma_{\omega}$ and an element a of $\mathscr{A}_{\omega} \backslash B$ s.t. $\rho$ cannot be extended to a partial lifting $\langle B \cup\{a\}\rangle \rightarrow \Sigma_{\omega}$.

Proof. Fix a measurable set $A \subseteq\{0,1\}^{\omega}$ s.t. both $A$ and $A^{c}$ have a dense set of points of density 1 (see [13]). Let $a=[A]$. Also fix a collection $\mathscr{C}=$ $\left\{N_{\alpha}: \alpha<\lambda\right\} \subseteq \mathscr{N}_{\omega}$ of subsets of $A^{c}$ s.t. $\bigcup \mathscr{C} \notin \mathscr{N}_{\omega}$. Inductively construct an increasing sequence $\left\langle B_{\alpha}: \alpha<\lambda\right\rangle$ of subalgebras of $\mathscr{A}_{\omega}$, a sequence $\left\langle E_{\alpha}: \alpha<\lambda\right\rangle$ of elements of $\Sigma_{\omega}$, and partial liftings $\left(\rho \mid B_{\alpha}\right): B_{\alpha} \rightarrow \Sigma_{\omega}$ satisfying

(0) For all $\alpha,\left|B_{\alpha}\right|<\lambda$;

(1) $B_{0}=\left\{[E]: E\right.$ is a clopen subset of $\left.\{0,1\}^{\omega}\right\} ; \rho([E])=E$ for all clopen $E$;

(2) $B_{\alpha+1}=\left\langle B_{\alpha} \cup\left\{e_{\alpha}\right\}\right\rangle, e_{\alpha}=\left[E_{\alpha}\right]$, and $\rho\left(e_{\alpha}\right)=E_{\alpha} \cup N_{\alpha}$; 
(3) If $\alpha$ is a limit ordinal, $B_{\alpha}=\bigcup_{\beta<\alpha} B_{\beta}, \rho\left|B_{\alpha}=\bigcup_{\beta<\alpha} \rho\right| B_{\beta}$; and for all $\alpha<\lambda$ and $x \in B_{\alpha}$ :

(4) If $x \neq 0$ then $x \wedge a \neq 0$;

(5) $x \leq a$ implies $\rho(x) \subseteq A \cup \cup_{\beta<\alpha} N_{\beta}$.

The initial and limit stages given by (1) and (3) present no difficulties ((4) and (5) hold at stage 0 by the choice of $A$ ).

Suppose now that $\rho \mid B_{\alpha}$ is given. Since $\left|B_{\alpha}\right|<\lambda, B_{\alpha} \mid a$ is not weakly dense in $\mathscr{A}_{\omega} \mid a$. Hence there is an $e_{\alpha}=\left[E_{\alpha}\right] \in \mathscr{A}_{\omega} \mid a\left(E_{\alpha} \subseteq A\right)$ s.t.

(6) For all $x \in B_{\alpha}$, if $x \neq 0$ then $e_{\alpha} \wedge x \wedge a \neq 0$ and $e_{\alpha}^{c} \wedge x \wedge a \neq 0$.

Extend $\rho$ to $\left\langle B_{\alpha} \cup\left\{e_{\alpha}\right\}\right\rangle=B_{\alpha+1}$ by letting $\rho\left(e_{\alpha}\right)=E_{\alpha} \cup N_{\alpha}((6)$ ensures that $\rho$ extends: see [14, Theorem 12.2] for example). We have to check (4) and (5) for $\alpha+1$.

Let $x \in B_{\alpha+1}$. Then for some $c, d \in B_{\alpha}, x=\left(e_{\alpha} \wedge c\right) \vee\left(d \backslash e_{\alpha}\right)$. If $x \neq 0$ then one of $c, d$ is not 0 . If $c \neq 0$ then by (6), $e_{\alpha} \wedge c \wedge a \neq 0$ and so $x \wedge a \neq 0$. Similarly if $d \neq 0$ then $x \wedge a \neq 0$ and so (4) holds. For (5), if $x \leq a$ then $d \leq a$ and $\rho(x) \subseteq \rho\left(e_{\alpha} \vee d\right)=\rho\left(e_{\alpha}\right) \cup \rho(d)=E_{\alpha} \cup N_{\alpha} \cup \rho(d) \subseteq A \cup \cup_{\beta<\alpha+1} N_{\beta}$. This completes the construction.

Now let $B=\bigcup_{\alpha<\lambda} B_{\alpha}, \rho=\bigcup_{\alpha<\lambda} \rho \mid B_{\alpha}$. (1) ensures that $\rho$ is a strong partial lifting. If $\rho$ could be extended to a partial lifting $\langle B \cup\{a\}\rangle \rightarrow \Sigma_{\omega}$, we would have $\bigcup \mathscr{C}=\bigcup_{\alpha<\lambda} N_{\alpha} \subseteq \bigcup_{\alpha<\lambda} \rho\left(e_{\alpha}\right) \subseteq \rho(a)$ but $\bigcup \mathscr{C}$ is a nonnull subset of $A^{c}$.

Remarks. (1) If $|B|<\lambda$ then $\rho$ extends: simply. let $\rho(a)=A \cup \bigcup\{\rho(b): b \leq a$ and $b \in B\} \backslash \bigcup\left\{\rho(b): b \leq a^{c}\right.$ and $\left.b \in B\right\}$.

(2) A similar argument can be used to get a subalgebra $B \subseteq \mathscr{A}_{\omega}$ of size $\aleph_{1}$ and a partial strong Borel lifting $\rho: B \rightarrow \Sigma_{\omega}$ (i.e. $\rho(a)$ is Borel for each $a \in B)$ which does not extend to a partial Borel lifting $\langle B \cup\{a\}\rangle \rightarrow \Sigma_{\omega}$.

(3) If we replace the condition that $\rho$ is strong by the stronger condition that $(\forall[E] \in B)\left(\rho(E) \supseteq\left\{x \in\{0,1\}^{\omega}: d(x, E)=1\right\}\right)$ then $\rho$ extends to a lifting $\mathscr{A}_{\omega} \rightarrow \Sigma_{\omega}$ regardless of the size of $B:$ for each $x \in\{0,1\}^{\omega}$ let $\mathscr{F}_{x}$ be an ultrafilter extending $\{[E]: d(x, E)=1\} \cup\{\rho(b): b \in B, x \in \rho(b)\}$. Define $\rho(a)=\left\{x \in\{0,1\}^{\omega}: a \in \mathscr{F}_{x}\right\}$ for all $a \in \mathscr{A}_{\omega}$. It is straightforward to check that this defines a lifting extending $\rho$.

Remark. W. Just has shown that GCH implies that the answer to the question in the introduction is no for ccc algebras. Also W. Weiss has shown that, in the notation of $(5)$ of the remarks in the introduction, there is a dense set of points $x \in \operatorname{St}(B)$ such that $\operatorname{wd}(B)=\pi \chi(x, \operatorname{St}(B))$. Thus the weak density of $B$ is the minimum $\pi$-character of a point in $\operatorname{St}(B)$.

\section{ACKNOWLEDGMENTS}

I would like to thank D. Fremlin, F. Tall, and W. Weiss for many helpful suggestions. 


\section{REFERENCES}

1. T. Carlson, $A$ theorem on lifting, undated handwritten notes.

2. J. Cichon, A. Kamburelis and J. Pawlikowski, On dense subsets of the measure algebra, Proc. Amer. Math. Soc. 94 (1985), 142-146.

3. E. van Douwen, Cardinal functions on Boolean spaces, Handbook of Boolean algebra, NorthHolland, Amsterdam (to appear).

4. D. Fremlin, On two theorems of Mokobodzki, note of June 23, 1977.

5. __ On additivity and cofinality of Radon measures, Mathematika 31 (1984), 323-335.

6. C. Goffman, Real functions, Prindle, Weber, and Schmidt, Boston, Mass., 1953.

7. P. Halmos, Measure theory, Van Nostrand, New York, 1950.

8. Hodel, Cardinal functions. I, Handbook of Set-Theoretic Topology (Kunen and Vaughan, eds.) North-Holland, 1984.

9. T. Jech, Set theory, Academic Press, 1978.

10. K. Kunen, Set theory, North-Holland, 1980.

11. M. Magidor, On the singular cardinals problem. I, Israel J. Math. 28 (1977), 1-31.

11a. D. Maharam, On homogeneous measure algebras, Proc. Nat. Acad. Sci. U.S.A. 28 (1942), 108-111.

12. J. Oxtoby, Measure and Category, Springer-Verlag, 1971.

13. W. Rudin, Well-distributed measurable sets, Amer. Math. Monthly 90 (1983), 41-42.

14. R. Sikorski, Boolean algebras, 2nd ed., Academic Press, 1964.

Department of Mathematics, University of Toronto, Toronto, Ontario M5S1al 